\title{
Long-term improvement of quality of life in patients with breast cancer: supporting patient-physician communication by an electronic tool for inpatient and outpatient care
}

\author{
Patricia Lindberg-Scharf ${ }^{1}\left[\right.$ (D) Brunhilde Steinger ${ }^{1} \cdot$ Michael Koller $^{2} \cdot$ Andrea Hofstädter $^{3} \cdot$ Olaf Ortmann $^{4} \cdot$ Jan Kurz $^{1}$. \\ Jonathan Sasse ${ }^{1} \cdot$ Monika Klinkhammer-Schalke $^{1}$
}

Received: 30 November 2020 / Accepted: 4 May 2021 / Published online: 27 June 2021

(c) The Author(s) 2021

\begin{abstract}
Purpose The effectiveness of a pathway with quality of life (QoL) diagnosis and therapy has been already demonstrated in an earlier randomized trial (RCT) in patients with breast cancer. We refined the pathway by developing and evaluating an electronic tool for QoL assessment in routine inpatient and outpatient care.

Methods In a single-arm study, patients with breast cancer with surgical treatment in two German hospitals were enrolled. QoL (EORTC QLQ-C30, QLQ-BR23) was measured with an electronic tool after surgery and during aftercare in outpatient medical practices (3, 6, 9, 12, 18, and 24 months) so that results (QoL-profile) were available immediately. Feedback by patients and physicians was analyzed to evaluate feasibility and impact on patient-physician communication.

Results Between May 2016 and July 2018, 56 patients were enrolled. Physicians evaluated the QoL pathway as feasible. Patients whose physician regularly discussed QoL-profiles with them reported significantly more often that their specific needs were cared for $(p<.001)$ and that their physician had found the right treatment strategy for these needs $(p<.001)$ compared with patients whose doctor never/rarely discussed QoL-profiles. The latter significantly more often had no benefit from QoL assessments $(p<.001)$.

Conclusion The QoL pathway with electronic QoL assessments is feasible for inpatient and outpatient care. QoL results should be discussed directly with the patient.
\end{abstract}

Clinical trial information NCT04334096, date of registration 06.04.2020

Keywords Quality of life · Patient-reported outcomes · Breast cancer · Electronic assessment · Patient-physician communication $\cdot$ Complex intervention

Patricia Lindberg-Scharf

patricia.lindberg-scharf@ur.de

1 Tumor Center Regensburg, Institute of Quality Management and Health Services Research of the University of Regensburg, Am Biopark 9, 93053 Regensburg, Germany

2 Center for Clinical Studies, University Hospital Regensburg, Franz-Josef-Strauß-Allee 11, 93053 Regensburg, Germany

3 Department of Obstetrics and Gynecology, University Hospital Regensburg, St. Hedwig Clinic, Steinmetzstraße 1-3, 93049 Regensburg, Germany

4 Department of Gynecology and Obstetrics, University Medical Center Regensburg, Landshuter Straße 65, 93053 RegensburgRegensburg, Germany

\section{Introduction}

Patients with cancer considerably suffer from impairments of their quality of life (QoL) during diagnosis, therapy, and aftercare. Hospitals more and more focus on additional supportive care (e.g. psycho-oncological care, nutritional therapy). However, patients usually stay in the hospital only for a few days whereas many QoL impairments appear in the later course of the disease during adjuvant therapy and aftercare $[1,2]$.

Studies have shown that structured QoL interventions for routine care are well accepted by patients and physicians [3-5], and improve patient-physician communication [6-8], QoL [7, 9], and even survival [9, 10]. A major limitation of these studies is that interventions were limited to the inpatient setting or to a single institution for outpatient care 
so that there is a lack of generalizability of these findings. Therefore, the Tumor Center Regensburg developed a QoL pathway with systematic QoL diagnosis and tailored QoL therapy for inpatient and outpatient care in a complex intervention $[11,12]$. Two randomized trials (RCTs) in patients with breast [13] and colorectal cancer [14] demonstrated effectiveness by showing a significantly better QoL in the intervention group patients. In both trials, QoL was measured with a paper-pencil questionnaire that was entered by hand into an electronic database to obtain results. Therefore, patients and physicians could discuss results only with a delay of about 1 week after measurement.

Electronic tools for the automatic assessment of patientreported outcomes have been developed, implemented, and evaluated in numerous studies [7-10]. Above all, such interventions have the advantage that results are available in real time for patients and physicians and can be directly discussed. However, there is a lack of feasibility for routine outpatient care because most medical practices do not have the necessary technical equipment for their patients such as tablet computers or computer workplaces.

Therefore, the aim of the present study was to overcome this problem by refining the QoL pathway so that QoL results could be electronically processed and presented in real time to patients and physicians in inpatient and outpatient care without the need for additional technical equipment such as tablet computers or smartphones in outpatient practices. Feasibility of the QoL pathway and its impact on patient-physician communication were analyzed.

\section{Methods}

\section{Study design}

The study was designed as a prospective, single-arm, clinical trial of a complex intervention including a one-group pretest-posttest design. Moreover, QoL data of the present study were compared with those of an earlier RCT [13] that investigated the effectiveness of the QoL pathway for patients with breast cancer.

\section{Participants and setting}

Patients were enrolled by study clinicians of two participating German Cancer Society (DKG)-certified breast cancer centers in Bavaria, Germany (Department of Gynecology and Obstetrics, University Medical Center Regensburg; Department of Obstetrics and Gynecology of the University Medical Regensburg, St. Hedwig Clinic). Inclusion criteria were diagnosis of breast cancer, with surgical treatment in one of the two hospitals, and informed consent to participate in the study. Exclusion criteria include the following: (1) recruiting study clinician unavailable; (2) patient misclassified in the operation schedule (no breast neoplasm); (3) coordinating practitioner refused trial participation; (4) no "Nachsorgekalendernummer" available (unique number of the diary a patient with cancer receives in the hospital for aftercare); (5) patient from district outside the defined study region; (6) age under 18 years; (7) pregnancy; (8) patient unable to fill out QoL questionnaires (physical, psychological, cognitive, language reasons); and (9) patient refused trial participation.

Aftercare was conducted in the outpatient practices of the patients' coordinating practitioners (CP: gynecologist responsible for aftercare). Patients were free to choose their CP. CPs of patients who agreed to participate were contacted by the recruiting clinician and asked to participate in the trial. The two study coordinators (PLS psychologist, BS gynecologist) individually trained each $\mathrm{CP}$ in an educational outreach visit in his/her practice by providing information about the aims and the procedure of the study. No additional study case-based payments were provided.

\section{Intervention}

All patients repeatedly filled out QoL questionnaires during the first 2 years after surgery. CPs received QoL results of their patients added by an address list with local healthcare professionals for specific QoL therapies. QoL data were automatically processed with the electronic data processing (EDP)-aided system "LPro":

\section{(1). Inpatient QoL diagnosis: tablet computer}

In the hospital, a clinician respectively study nurse conducted the first QoL measurement (baseline) when the patient came for routine consultation 1 week after discharge. Patients answered the QoL questionnaire on a tablet computer. A presentation of one question per screen was chosen to allow for better focus and the duration of time to fill out the questionnaire was automatically recorded. A final question asked for technical problems. After finishing data entry, a QoL profile (see Fig. 1) with results was displayed on the screen so that the clinician could discuss the profile immediately with the patient. Simultaneously, the profile was sent via e-mail to the authorized clinicians of the hospital and the study coordinators. Alternatively, patients could also complete a paper-pencil version of the QoL questionnaire based on preference as studies have shown that results of electronic and paper-pencil measurements are equivalent $[15,16]$. However, in this case the QoL-profile was not available in realtime.

(2). Outpatient QoL diagnosis: EDP-aided paper-based assessment 
Fig. 1 Quality of life (QoL-) profile: woman with primary breast cancer, 49 years, married, one child, working. Prognostic classification $\mathrm{pT} 1 \mathrm{~b}(\mathrm{~m}), \mathrm{SN} 0$, M0, G1, ER pos, PR pos, HER2 neg; breast-conserving surgical therapy with revision surgery after 1 month followed by radiation and anti-estrogen treatment. Red bar $=$ cutoff for a need for QoL therapy $(<50$ points)

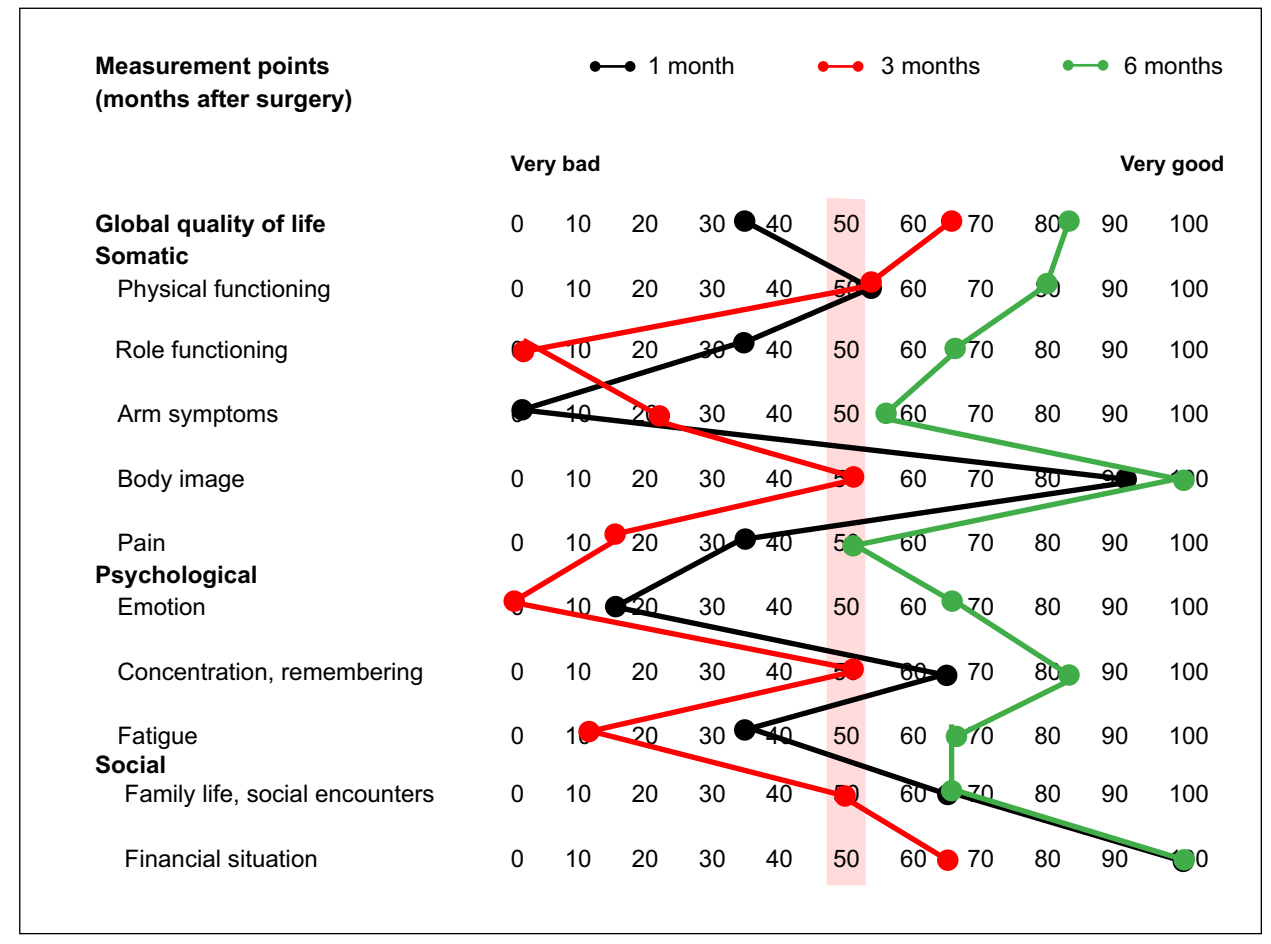

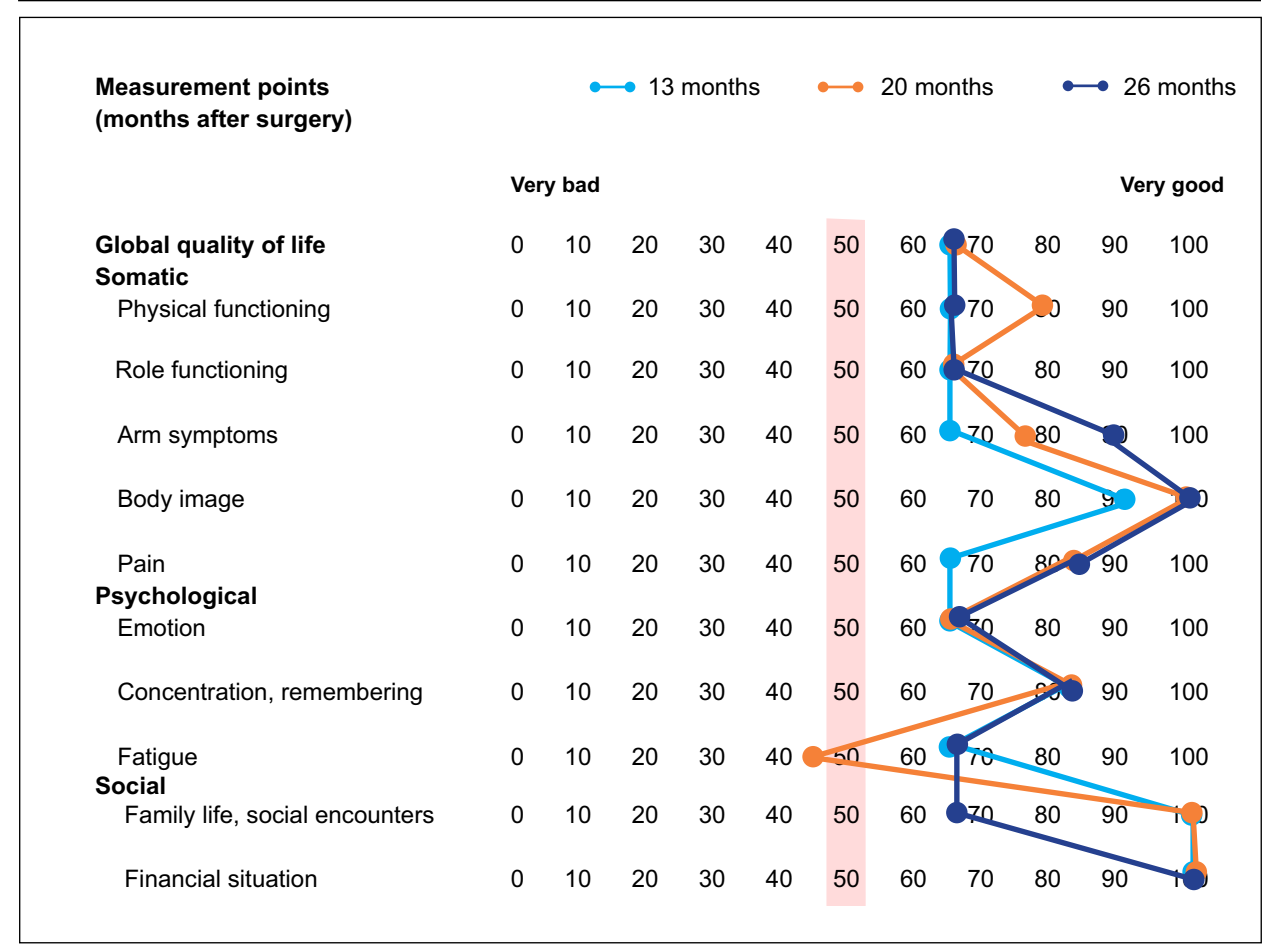

Further QoL measurements were conducted in the practice of the $\mathrm{CP}$ in accordance with the national practice guideline for breast cancer at 3, 6, 9, 12, 18, and 24 months [17]. Usually, outpatient practices do not have a tablet computer or computer workplace for patients. Therefore, an EDPaided paper-based method was developed (see Supplementary Fig.): CPs received a paper-pencil version of the QoL questionnaire. An individual QR code with pseudonymized patient information was printed on each questionnaire ensuring that QoL data were assigned to the right patient. For each assessment time point, participants received a postal reminder. The questionnaire was given to the patient during her visit in the practice to be completed during waiting. After that, the questionnaire was sent to a local digital fax server where it was automatically processed and answers were stored in a database. Following this, a QoL-profile 
was generated and transferred back to the CP's practice via e-mail or fax (depending on CP's preference). The processing time took on average $17.43 \mathrm{~s}(\mathrm{SD}=23.17)$. In the Supplementary Information, a detailed description of server configuration and used software can be found. The immediate response enabled patients and CPs to discuss the QoLprofile directly. If a patient`s QoL was below 50 points in at least one dimension of the QoL profile the $\mathrm{CP}$ automatically received an address list with local healthcare providers of a multiprofessional care network.

\section{Multiprofessional network for inpatient and outpatient care}

For the tailored treatment of QoL, a regional network structure of healthcare providers for inpatient and outpatient care had already been established during the two earlier RCTs $[13,14]$. This care network encompassed a total of 54 therapists providing the following different therapeutic options:

- physiotherapy $(n=9)$,

- psychotherapy $(n=12)$,

- pain therapy $(n=6)$,

- social support $(n=6)$,

- nutritional counseling $(n=14)$,

- fitness $(n=7)$

For each therapeutic option, therapists regularly met in quality circles. All professionals were certified in their respective field (e.g., certified psycho-oncologist or certified stoma nurse). CPs received contact information of quality circle members to contact them for QoL therapy.

\section{Measures}

\section{Quality of life}

Quality of life was measured with the European Organization for Research and Treatment of Cancer (EORTC) core questionnaire QLQ-C30 (version 3.0) [18] and the breast cancer-specific module EORTC QLQ-BR23 [19]. EORTC QLQ-C30 aggregates 30 items into six functioning scales, three symptom scales, and five single items. The EORTC QLQ-BR23 consists of 23 items that are aggregated into two functional scales, three symptom scales, and three single items. All scores were linearly transformed and presented on scales from 0 to 100 [20]. We used a uniform manner for transformation with 0 denoting the negative (low functioning, high symptom burden) and 100 the positive end (high functioning, low symptom burden) of the continuum [21]. The QoL profile (see Fig. 1) shows a patient's QoL on eleven scales of the QLQ-C30 and QLQ-BR23. We had selected these scales based on the experience of the previous RCT [13], appraisal of relevance, and the availability of specific therapies to improve QoL. A cutoff score $<50$ points defined a "need for QoL therapy". This decision criterion was chosen to highlight the patient's perspective of subjective wellbeing by dichotomizing symptom scores with a majority of "quite a bit" and "very much" responses to the "bad" side of the spectrum $(<50)$ and "not at all" and "a little" responses to the "good" side $(\geq 50)[13,22,23]$

\section{Health status}

The recruiting clinician documented the following demographic and clinical patient variables at study entry: age, marital status, employment status, number of children, tumor stage, primary disease, receptor status, date of surgery, surgical procedure, comorbidities, and neoadjuvant therapy.

\section{Patient evaluation}

At 6 and 24 months after surgery, patients received a paperbased self-developed questionnaire with quantitative questions asking for feedback about the usefulness of the QoL pathway from their point of view.

\section{Physician evaluation}

At 24 months postoperatively, CPs received a paper-based self-developed questionnaire with quantitative questions asking for feedback about the feasibility, acceptability, and usefulness of the QoL pathway.

The management and storage of data were in accordance with the European General Data Protection Regulation (GDPR). The trial was approved by the ethics committee of the University of Regensburg (reference number 15-101-0320).

\section{Statistical analysis}

All statistical comparisons were two-tailed and a $p$ value of $<0.05$ indicated statistical significance. Continuous variables are presented as means (standard deviation) and categorical variables as absolute and relative frequencies. A need for QoL therapy was defined as a score $<50$ points in at least one of eleven dimensions of the QoLprofile. Rates of patients with a need for QoL therapy and rates of patients with a score $<50$ points in each single QoL dimension were compared for 0 and 6 months with McNemar tests. This timepoint was chosen as it has been the primary endpoint in the previous RCT [13]. Rates of patients with a need for QoL therapy in the present study 
were compared with rates of two historical controls of this RCT (namely the RCT`s intervention and control group) with $\chi^{2}$ tests. For these comparisons, the scale "financial functioning" was excluded because this scale was not part of the RCT. All analyzes were performed using SPSS version 25.0 (IBM Corp., Armonk, NY, USA).

\section{Results}

Between 30 May 2016 and 18 July 2018, 88 patients who fulfilled inclusion criteria were invited to participate in the trial. Of those, $56(64 \%)$ agreed and were included in the study. Table 1 shows baseline characteristics of the study sample. Patients who refused $(n=32)$ were significantly older (mean age 66.50 vs. $50.63, p<0.001$ ), and the mastectomy rate was significantly higher $(36 \%$ vs. $9 \%, p=0.002)$. All participants provided written informed consent.

\section{Questionnaire completion mode}

In the hospital, $82 \%$ (46/56) of participants completed the first QoL measurement on the tablet computer whereas $14 \%$ $(8 / 56)$ preferred the paper-based questionnaire (unknown $4 \%, 2 / 56)$. The mean time for completion of the QoL questionnaire on the tablet computer was $9.9 \mathrm{~min}(\mathrm{SD}=3.4 \mathrm{~min}$, range 4.9-19.0 $\mathrm{min}$ ). Of those patients who used the tablet computer, $91 \%$ (42/46) reported no technical problems, $7 \%(3 / 46)$ a little, and $2 \%(1 / 46)$ quite a bit. No participant had serious technical problems. However, 39\% (18/46) of women were supported by medical staff when entering data for unknown reasons. Those patients did not differ in their mean age (51.6 years) from women who had no support for questionnaire completion (50.5 years) or who preferred the paper-based questionnaire (52.0 years) $(p=0.421)$. During aftercare $75 \%$ of QoL questionnaires were transferred by fax, $23 \%$ by post, and $2 \%$ were completed on the tablet computer in the hospital.

\section{Questionnaire completion rates}

Figure 2 shows questionnaire completion rates during the 24-month study period. After 12 months, two women refused further trial participation because they felt well with no need for QoL measurements anymore. Seven patients were lost to follow up during the first year of the study and six during the second year. During aftercare the questionnaire completion rate was lowest at 3 months $(n=29)$ and highest at 6 months $(n=41)$. The last QoL measurement at 24 months was completed by 34 women.
Table 1 Baseline characteristics of participants at study entry

\begin{tabular}{|c|c|}
\hline & $\begin{array}{l}\text { No. }(\%) \text { of } \\
\text { participants } \\
(n=56)\end{array}$ \\
\hline Age (years) mean (SD) & $50.63(9.68)$ \\
\hline \multicolumn{2}{|l|}{ Marital status $n(\%)$} \\
\hline Married & $35(63)$ \\
\hline Unmarried & $10(18)$ \\
\hline Divorced & $4(7)$ \\
\hline Separated & $2(4)$ \\
\hline Widowed & $2(4)$ \\
\hline Unknown & $3(5)$ \\
\hline \multicolumn{2}{|l|}{ Children $n(\%)$} \\
\hline Yes & $39(70)$ \\
\hline No & $11(20)$ \\
\hline Unknown & $6(11)$ \\
\hline \multicolumn{2}{|l|}{ Employment status $n(\%)$} \\
\hline Employed & $30(54)$ \\
\hline Retired/not employed & $26(46)$ \\
\hline \multicolumn{2}{|l|}{ Prognostic stage at diagnosis $n(\%)$} \\
\hline UICC 0 & $1(2)$ \\
\hline UICC I & $21(38)$ \\
\hline UICC II & $27(48)$ \\
\hline UICC III & $6(11)$ \\
\hline UICC IV & $1(2)$ \\
\hline \multicolumn{2}{|l|}{ Primary disease $n(\%)$} \\
\hline Yes & $54(96)$ \\
\hline No & $2(4)$ \\
\hline \multicolumn{2}{|l|}{ Surgical procedure $n(\%)$} \\
\hline Breast conserving therapy & $51(91)$ \\
\hline Mastectomy & $5(9)$ \\
\hline Estrogen receptor positive $n(\%)$ & $45(80)$ \\
\hline Progesterone receptor positive $n(\%)$ & $44(79)$ \\
\hline HER2 positive $n(\%)$ & $9(16)$ \\
\hline \multicolumn{2}{|l|}{ Comorbidities $n(\%)$} \\
\hline Cardiovascular & $6(11)$ \\
\hline Lung & $5(9)$ \\
\hline Kidney & $1(2)$ \\
\hline Central nervous system & $2(4)$ \\
\hline \multicolumn{2}{|l|}{ Neoadjuvant therapy $n(\%)$} \\
\hline Chemotherapy & $14(25)$ \\
\hline None & $39(70)$ \\
\hline Unknown & $3(5)$ \\
\hline
\end{tabular}

\section{Participation of CPs}

In total, 34 CPs were approached of whom 33 (97\%) participated in the trial. Of those, one CP dropped out during the course of the study due to a lack of time. 


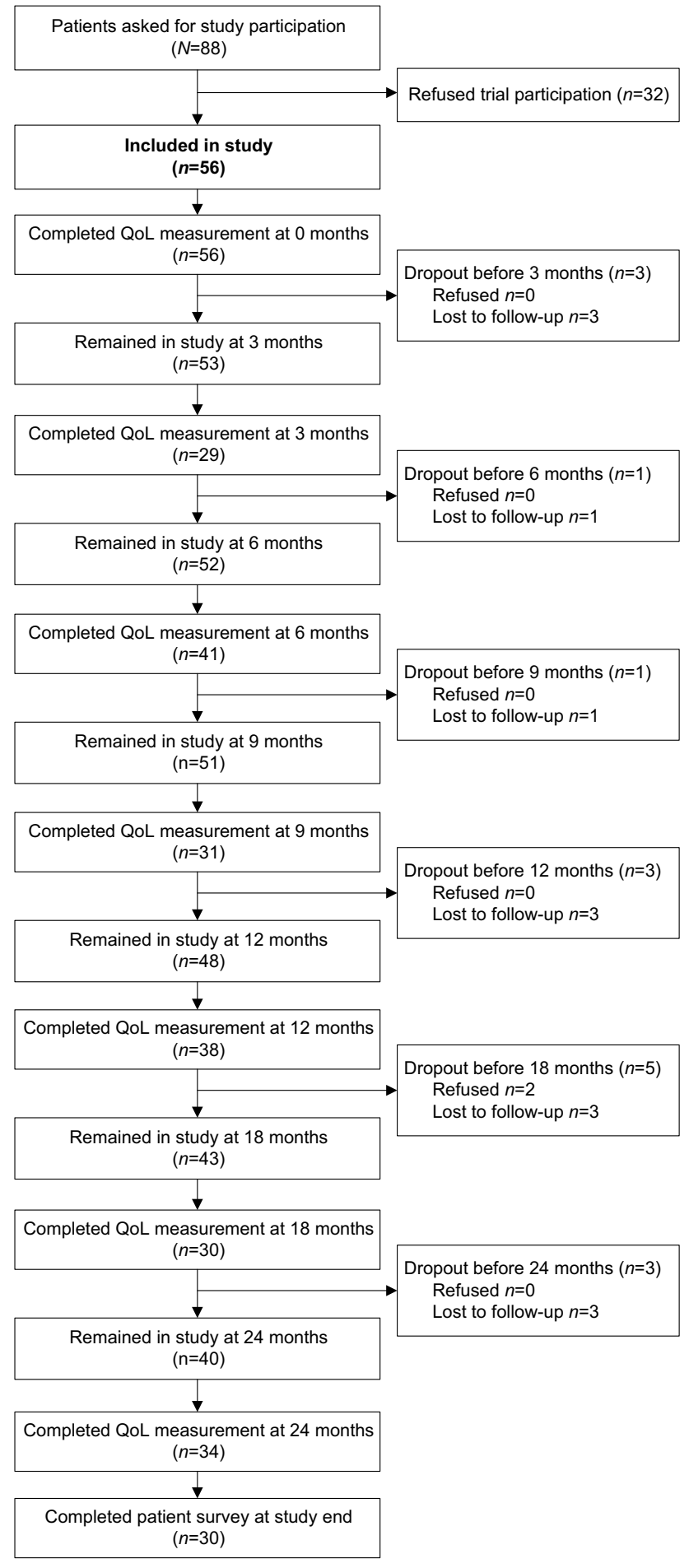

Fig. 2 Flow chart

\section{Rates of patients with a need for QoL therapy}

A comparison of rates of patients with a need for QoL therapy at 0 and 6 months postoperatively revealed a non-significant reduction from $70 \%(28 / 40)$ to $63 \%(25 / 40)(p=0.581)$. In single QoL dimensions, rates significantly decreased in
Table 2 Comparison of rates of patients with a need for QoL therapy at 0 and 6 months after surgery

\begin{tabular}{llll}
\hline & 0 months & 6 months & $\boldsymbol{p}^{\mathbf{c}}$ \\
\hline Total $^{\mathrm{a}}$ & $70 \%$ & $63 \%$ & .58 \\
Single QoL dimensions $^{\mathrm{b}}$ & & & \\
$\quad$ Global QoL & $29 \%$ & $22 \%$ & .61 \\
$\quad$ Physical functioning & $7 \%$ & $5 \%$ & 1.00 \\
Role functioning & $34 \%$ & $29 \%$ & .79 \\
Pain & $32 \%$ & $24 \%$ & .58 \\
Body image & $12 \%$ & $20 \%$ & .38 \\
Arm symptoms & $38 \%$ & $15 \%$ & $\mathbf{. 0 1}$ \\
Emotional functioning & $29 \%$ & $34 \%$ & .75 \\
Cognitive functioning & $12 \%$ & $22 \%$ & .29 \\
Fatigue & $34 \%$ & $44 \%$ & .34 \\
Social functioning & $22 \%$ & $25 \%$ & 1.00 \\
Financial impact & $8 \%$ & $28 \%$ & $\mathbf{. 0 1}$ \\
\hline
\end{tabular}

${ }^{\mathrm{a}}$ Need for QoL therapy (QoL $<50$ points) in at least one of eleven QoL dimensions of EORTC QLQ-C30, QLQ-BR23

${ }^{\mathrm{b}}$ Need for QoL therapy (QoL $<50$ points) in single QoL dimensions of EORTC QLQ-C30, QLQ-BR23

${ }^{\mathrm{c}}$ All $p$ values derived from McNemar tests $p$ values $<0.05$ are presented in bold face

the dimension "arm symptoms" (38\% vs. $15 \%, p=0.01)$ and increased in the dimension "financial impact" (8\% vs. $28 \%$, $p=0.01)$ at 6 months. On the other scales, there were no significant differences between 0 and 6 months (see Table 2).

Moreover, QoL data during the first 12 months after surgery were compared with those of the former RCT's intervention $(n=99)$ and control group $(n=100)$ [13]. Participants of the present study (mean age 50.63 years, $\mathrm{SD}=9.68$ ) were significantly younger than women of both historical controls (RCT's intervention group 58.63 years, $\mathrm{SD}=12.09$; RCT's control group 56.75 years, $\mathrm{SD}=11.92$, $F=8.84, p<0.001)$ with a higher rate of breast conserving therapy (91\% vs. RCT`s intervention group $75 \%$ vs. RCT`s control group 76\%; $p=0.039$ ). As shown in Fig. 3, rates of patients with a need for QoL therapy in the present sample were comparable with those of the RCT's intervention group and consistently lower than in the RCT's control group. Subgroup analysis comparing rates of patients with a need for QoL therapy in the present sample with both historical controls revealed no significant differences at the primary endpoint at 6 months.

\section{Patient evaluation}

Patients were asked via questionnaire to evaluate the $\mathrm{QoL}$ pathway at 6 and 24 months after surgery. The completion rates were $70 \%$ (6 months: $39 / 56$ ) respectively $54 \%$ (24 months: 30/56). At 6 months, 74\% (29/39) of patients 


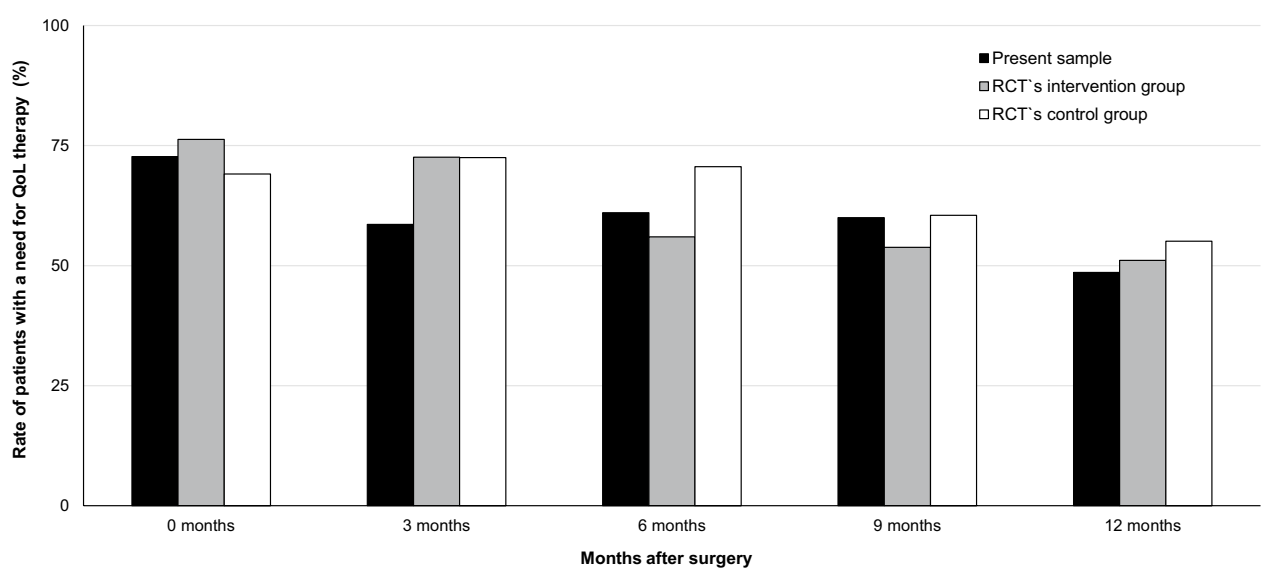

Fig. 3 Rates of patients with a need for quality of life (QoL) therapy ( $\mathrm{QoL}<50$ points on at least one of ten scales) in the present sample compared with two historical controls of the previous RCT (namely RCT's intervention and control group) [13] over 12 months; scale "financial functioning" was excluded from the analyzes because this

reported that their CP had discussed QoL-profiles with them whereas in $26 \%$ (10/39), this happened never or only rarely. At 24 months, the QoL-profile had been discussed with $60 \%(18 / 30)$ of participants compared to $40 \%(12 / 30)$ whose $\mathrm{CP}$ had never or only rarely discussed profiles with them. Table 3 shows results of patient evaluations at 6 and 24 months comparing both subgroups.

Six months postoperatively significantly more women whose CP regularly discussed QoL-profiles with them reported that the discussion was helpful $(83 \%$ vs. $0 \%$, $p<0.001)$ and that their specific needs like pain, sorrows, or anxiety had also been cared for $(72 \%$ vs. $30 \%, p=0.04)$ in comparison to patients whose $\mathrm{CP}$ never or only rarely discussed QoL-profiles. At 24 months, regular discussions of QoL-profiles were associated with a higher proportion of women who evaluated these discussions as helpful ( $78 \%$ vs. $8 \%, p<0.001)$ and who rated the dimensions of the QoLprofile as personally relevant ( $94 \%$ vs. $33 \%, p=0.01)$. They also reported significantly more often that their wellbeing had been discussed more often during the medical appointment $(61 \%$ vs. $8 \%, p<001)$, that their specific needs had been cared for more frequently ( $94 \%$ vs. $25 \%, p<0.001$ ), and that the right treatment strategy had been found $(89 \%$ vs. $17 \%, p<0.001)$. In contrast, significantly more women whose CP rarely or never discussed QoL-profiles with them reported no benefit by answering QoL questionnaires at $6(14 \%$ vs. $60 \%, p=0.01)$ and 24 months $(6 \%$ vs. $77 \%$, $p<0.001)$.

\section{Physician evaluation}

The physician evaluation at 24 months postoperatively was answered by $52 \%(17 / 33)$ of CPs. Of those, $94 \%(16 / 17)$ scale was not part of the RCT; rates at 18 and 24 months are not analyzed because these were not assessed in the RCT; $\chi^{2}$ tests: 0 months $p=.54,3$ months $p=.32$, 6 months $p=.14,9$ months $p=.66$, 12 months $p=.77$

found that the QoL pathway was useful for patients with breast cancer with $82 \%(14 / 17)$ reporting that it met the needs of their patients. In detail, 94\% (16/17) of CPs discussed the QoL-profile with their patients, $88 \%(15 / 17)$ evaluated it as helpful, and $82 \%(14 / 17)$ reported that the profile had improved patient-physician communication. The dimensions of the QoL-profile were rated as appropriate by all CPs (17/17). Moreover, the address list with QoL therapists was useful for all CPs (17/17) and 94\% (16/17) evaluated the EDP-aided paper-based assessment of QoL as feasible. Seventy-six percent (13/17) of CPs wanted to receive the QoL-profile for their other patients with cancer as well.

To assess for bias in the physician survey, we analyzed if CPs whose patients had reported at 6 months in the patient survey that their CP regularly discussed QoL-profiles with them were more likely to participate in the physician survey compared with those CPs who rarely/never discussed QoL-profiles. In total, $26 \mathrm{CPs}$ treated the 39 patients who responded to the patient evaluation at 6 months and 13 of those participated in the physician survey. Participation rates in the physician survey did not differ significantly between CPs who regularly discussed QoL-profiles $(9 / 19,47 \%)$ and CPs who rarely/never discussed QoL-profiles (4/7, 57\%; $p=1.00)$.

\section{Discussion}

The refined QoL pathway including the electronic tool LPro to present QoL results immediately after measurement was shown to be feasible and useful for inpatient and outpatient care of patients with breast cancer. The vast majority of CPs who participated in the physician 
Table 3 Patient evaluations at 6 and 24 months after surgery: subgroup analyzes of participants whose CP "regularly" versus "rarely or never" discussed QoL-profiles with them

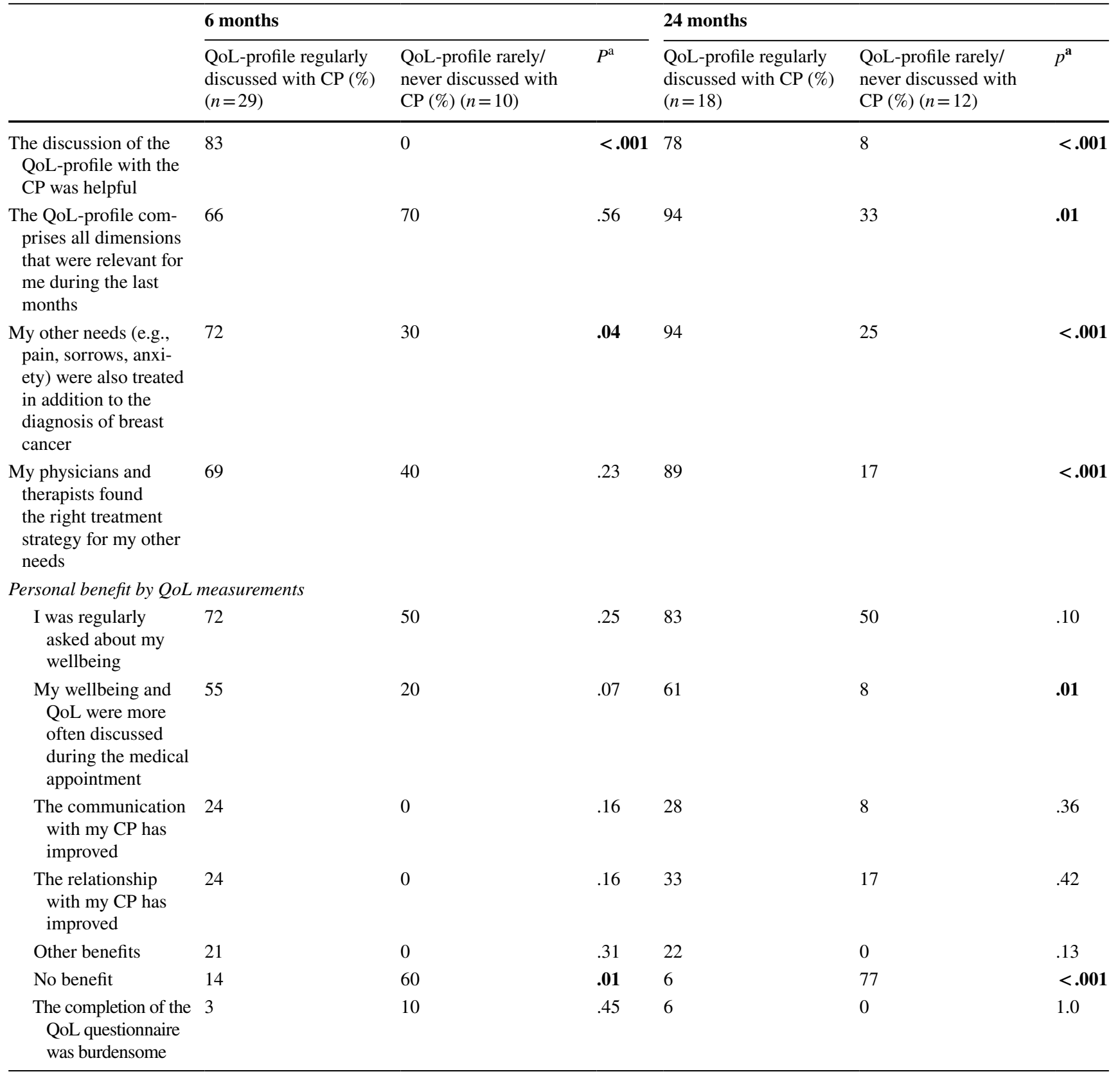

${ }^{\text {a All }} p$ values derived from $\chi^{2}$ tests or Fisher's exacts test if smallest expected cell value was $<5$

$p$ values $<0.05$ are presented in bold face

evaluation reported that the pathway met patients' needs and improved patient-physician communication what is in line with previous studies [6-8]. Participation rates of CPs support this finding as only one $\mathrm{CP}$ refused participation and another CP dropped out during the study. It was interesting that most CPs preferred to receive the QoL-profile via fax whereas one $\mathrm{CP}$ wanted to receive profiles by post and none by e-mail. It has been shown that compliance in practice is higher if fewer new skills and organizational change are needed [24]. Accordingly, new (technical) interventions must be kept as simple as possible for the implementation into the workflow of routine care.

Another important finding is that patients also subjectively benefited from QoL measurements given that their physician discussed QoL results with them. In our study all CPs were recommended to use QoL-profiles for patient-physician communication but a considerable part of patients reported that this happened only rarely or never. A systematic review [25] 
identified an imbalance in the patient-physician relationship as a potential barrier for the use of patient-reported outcomes that is among others caused by interpersonal characteristics of the doctor like a mere focus on treating a "disease" or dominating decision-making by an authoritarian behavior. It is also possible that CPs considered QoL-profiles during decision-making but did not discuss them directly with their patients. Indeed, discussing QoL results is very important for patients. Thus, we found that participants whose CP regularly discussed QoL-profiles with them evaluated this as helpful and reported that their specific needs were cared for more often. In contrast, patients whose CP never or only rarely discussed QoL-profiles with them significantly more often had no subjective benefit of QoL measurements. This result demonstrates that it is important to integrate QoL diagnosis and therapy into the workflow of aftercare so that QoL can be directly considered and discussed during the medical encounter. QoL measurements at the patient's home (e.g., via mobile app) that are not discussed with the physician are no adequate alternative. This finding is also important to encourage doctors to use QoL results in patient-physician communication.

Results of the earlier RCT [13] have demonstrated that patients who received QoL diagnosis and therapy showed a faster improvement of their QoL during the first year after surgery. In our sample, rates of patients with a need for $\mathrm{QoL}$ therapy were comparable with those of former intervention group patients and consistently lower than in the former control group of the RCT. Differences between groups were not significant because of the small sample size but showed a consistent trend.

In contrast to the earlier RCT [13], patients in the present study did not receive any further reminder if they missed a QoL measurement and there was no regular phone contact between study coordinators and CPs. This resulted in lower follow-up rates in the present sample. However, at the end of the trial at 24 months there were still $71 \%$ of participants in the study. Thus, the majority of patients used QoL diagnosis and therapy throughout the first 2 years after surgery. Some patients dropped out during the course of the study because they felt good and had no further need for QoL therapy. The duration of the QoL pathway should be individually adapted based on medical assessment and patients' preferences.

The study also has some limitations. First, the sample size was relatively small. Initially, a sample size of 200 patients was planned according to the previous RCT with 200 participants [13]. Because of logistical reasons, we decided to include only two regional hospitals that were responsible for patient recruitment in the present study compared with five recruiting hospitals in the RCT so that the sample size of 200 patients could not be reached within the recruitment period. A second limitation was that $36 \%$ of patients refused to participate in the study compared with only $18 \%$ in the RCT. The specific components of the QoL pathway were identical in both studies (QoL diagnosis and therapy) except of using a tablet computer for the first QoL measurement. This could have reduced the acceptability of the intervention. Participants of the present study were significantly younger compared with RCT patients and with women who refused participation. Wysham et al. [26] found a higher age to be a significant predictor of lower completion rates of electronic patient-reported outcomes in routine cancer care. We tried to address this problem by also offering a paper-pencil version of the questionnaire that was used by $14 \%$ of participants. This shows that there needs to be a paper-based alternative to electronic QoL assessments so that more patients can be reached. Furthermore, patient characteristics of historical controls were not completely comparable with those of patients in the present sample (e.g., age, surgical procedure). This may have caused a bias when comparing rates of patients with a need for QoL therapy. In addition, results of the physician survey may be biased by the response rate of 52\%. A subgroup analysis showed that participation rates of the physician survey did not differ between CPs who regularly discussed QoL-profiles and those who rarely/never discussed profiles. Thus, highly motivated CPs were not more likely to participate in the survey. Finally, because of multiple testing, results need to be confirmed in future trials.

To our knowledge, this is the first study providing results of QoL measurements immediately to patients and their physicians in a broad inpatient and outpatient setting with a relatively low-tech technical equipment, other than tablet computers or smartphones. In conclusion, patients with breast cancer subjectively benefit from QoL diagnosis and therapy in respect of a higher satisfaction with care given that their doctor discusses QoL results with them. Two previous RCTs $[13,14]$ have already demonstrated effectiveness of QoL diagnosis and therapy in terms of a better QoL in patients with breast cancer and colorectal cancer. Therefore, QoL diagnosis and therapy should be offered to all cancer patients during treatment and aftercare and regular funding needs to be established, e.g., by health insurances.

Supplementary Information The online version contains supplementary material available at https://doi.org/10.1007/s00520-021-06270-1.

Acknowledgements The study was funded by German Cancer Aid (Deutsche Krebshilfe, grant no. 111169). The authors would like to thank the study participants, the two recruiting breast cancer centers, and the many coordinating practitioners and healthcare professionals who supported the trial. We are grateful to the late Prof. Wilfried Lorenz ( $\mathrm{f} 2014$ ) for his expertise in the development of the study conception and design. We also acknowledge the contributions of the late Dr. Brigitte Ernst (f 2019) to the evolution of the Tumor Center Regensburg and to patient-centered oncological care. 
Author contributions All authors contributed to the study conception and design. AH, PLS, OO, and BS were involved in the collection of data. MKS, JK, PLS, JS, and BS contributed to the development of the software LPro and PLS was responsible for data analysis. All authors contributed to the interpretation of the data, the preparation and writing of the manuscript, and approved the final manuscript.

Funding Open Access funding enabled and organized by Projekt DEAL. The study was funded by German Cancer Aid (Deutsche Krebshilfe, grant no. 111169).

Data availability The authors have full control of all primary data and agree to allow the journal to review the data if requested.

Code availability Not applicable.

\section{Declaration}

Ethics approval The trial was approved by the ethics committee of the University of Regensburg (reference number 15-101-0320) and was performed in line with the principles of the Declaration of Helsinki.

Consent to participate All participants provided written informed consent.

Consent for publication Not applicable.

Conflict of interest The authors declare that they have no conflict of interest.

Open Access This article is licensed under a Creative Commons Attribution 4.0 International License, which permits use, sharing, adaptation, distribution and reproduction in any medium or format, as long as you give appropriate credit to the original author(s) and the source, provide a link to the Creative Commons licence, and indicate if changes were made. The images or other third party material in this article are included in the article's Creative Commons licence, unless indicated otherwise in a credit line to the material. If material is not included in the article's Creative Commons licence and your intended use is not permitted by statutory regulation or exceeds the permitted use, you will need to obtain permission directly from the copyright holder. To view a copy of this licence, visit http://creativecommons.org/licenses/by/4.0/.

\section{References}

1. Wöckel A, Schwentner L, Krockenberger M, Kreienberg R, Janni W, Wischnewsky M, Thorsten K, Felix F, Riccardo F, Blettner M, Singer S (2017) Predictors of the course of quality of life during therapy in women with primary breast cancer. Qual Life Res 26(8):2201-2208. https://doi.org/10.1007/s11136-017-1570-0

2. Couwenberg AM, Burbach JPM, van Grevenstein WMU, Smits AB, Consten ECJ, Schiphorst AHW, Wijffels NAT, Heikens JT, Intven MPW, Verkooijen HM (2018) Effect of neoadjuvant therapy and rectal surgery on health-related quality of life in patients with rectal cancer during the first 2 years after diagnosis. Clin Colorectal Cancer 17(3):e499-e512. https://doi.org/10.1016/j. clcc.2018.03.009

3. Detmar SB, Aaronson NK, Wever LDV, Muller M, Schornagel JH (2000) How are you feeling? Who wants to know? Patients' and oncologists' preferences for discussing health-related quality-of-life issues. J Clin Oncol 18:3295-3301. https://doi. org/10.1200/JCO.2000.18.18.3295

4. Braeken APBM, Kempen GIJM, Eekers D, van Gils FCJM, Houben RMA, Lechner L (2011) The usefulness and feasibility of a screening instrument to identify psychosocial problems in patients receiving curative radiotherapy: a process evaluation. BMC Cancer 11:479. https://doi.org/10.1186/1471-2407-11-479

5. Cleeland CS, Wang XS, Shi Q, Mendoza TR, Wright SL, Berry MD, Malveaux D, Shah PK, Gning I, Hofstetter WL, Putnam JB Jr, Vaporciyan AA (2011) Automated symptom alerts reduce postoperative symptom severity after cancer surgery: a randomized controlled clinical trial. J Clin Oncol 29:994-1000. https://doi. org/10.1200/JCO.2010.29.8315

6. Detmar S, Muller M, Schornagel J, Wever LDV, Aaronson NK (2002) Health-related quality-of-life assessments and patientphysician communication A randomized controlled trial. JAMA 288:3027-3034. https://doi.org/10.1001/jama.288.23.3027

7. Velikova G, Booth L, Smith AB, Brown PM, Lynch P, Brown JM, Selby PJ (2004) Measuring quality of life in routine oncology practice improves communication and patient well-being: a randomized controlled trial. J Clin Oncol 22:714-724. https://doi. org/10.1200/JCO.2004.06.078

8. Berry DL, Blumenstein BA, Halpenny B, Wolpin S, Fann JR, Austin-Seymour M, Bush N, Karras BT, Lober WB, McCorkle R (2011) Enhancing patient-provider communication with the electronic self-report assessment for cancer: a randomized trial. J Clin Oncol 29:1029-1035. https://doi.org/10.1200/JCO.2010.30.3909

9. Basch E, Deal AM, Kris MG, Scher HI, Hudis CA, Sabbatini P, Rogak L, Bennett AV, Dueck AC, Atkinson TM, Chou JF, Dulko D, Sit L, Barz A, Novotny P, Fruscione M, Sloan JA, Schrag D (2016) Symptom monitoring with patient-reported outcomes during routine cancer treatment: a randomized controlled trial. J Clin Oncol 34:557-5565. https://doi.org/10.1200/JCO.2015.63.0830

10. Denis F, Lethrosne C, Pourel N, Molinier O, Pointreau Y, Domont J, Bourgeois H, Senellart H, Trémolières P, Lizée T, Bennouna J, Urban T, El Khouri C, Charron A, Septans AL, Balavoine M, Landry S, Solal-Céligny P, Letellier C (2017) Randomized trial comparing a web-mediated follow-up with routine surveillance in lung cancer patients. JNCI J Natl Cancer Inst 109(9). https://doi. org/10.1093/jnci/djx029

11. Campbell M, Fitzpatrick R, Haines A, Kinmonth AL, Sandercock P, Spiegelhalter P, Tyrer P (2000) Framework for design and evaluation of complex interventions to improve health. BMJ 321:694-696. https://doi.org/10.1136/bmj.321.7262.694

12. Craig P, Dieppe P, Macintyre S, Mitchie S, Nazareth I, Petticrew M (2008) Developing and evaluating complex interventions: the new Medical Research Council guidance. BMJ 337:979-983. https://doi.org/10.1136/bmj.a1655

13. Klinkhammer-Schalke M, Koller M, Steinger B, Ehret C, Ernst B, Wyatt JC, Hofstädter F, Lorenz W (2012) Direct improvement of quality of life using a tailored quality of life diagnosis and therapy approach: randomized trial in 200 women with breast cancer. $\mathrm{Br}$ J Cancer 106:826-838. https://doi.org/10.1038/bjc.2012.4

14. Klinkhammer-Schalke M, Steinger B, Koller M, Zeman F, Fürst A, Gumpp J, Obermaier R, Piso P, Lindberg-Scharf P (2020) Diagnosing deficits in quality of life and providing tailored therapeutic options: results of a randomised trial in 220 patients with colorectal cancer. Eur J Cancer 130:102-113. https://doi.org/10. 1016/j.ejca.2020.01.025

15. Gwaltney CJ, Shields AL, Shiffman S (2008) Equivalence of electronic and paper-and-pencil administration of patient-reported outcome measures: a meta-analytic review. Value Health 11:32233. https://doi.org/10.1111/j.1524-4733.2007.00231.x

16. Muehlhausen W, Doll H, Quadri N, Fordham B, O'Donohoe P, Dogar N, Wild DJ (2015) Equivalence of electronic and paper administration of patient-reported outcome measures: a systematic 
review and meta-analysis of studies conducted between 2007 and 2013. Health Qual Life Outcomes 13:167. https://doi.org/10.1186/ s12955-015-0362-x

17. Leitlinienprogramm Onkologie (Deutsche Krebsgesellschaft, Deutsche Krebshilfe, AWMF) (2020) S3-Leitlinie Früherkennung, Diagnose, Therapie und Nachsorge des Mammakarzinoms, Version 4.3. AWMF Registernummer: 032-045OL. http://www. leitlinienprogramm-onkologie.de/leitlinien/mammakarzinom/. Accessed 27 April 2020

18. Aaronson NK, Ahmedzai S, Bergman B et al (1993) The European Organization for Research and Treatment of Cancer QLQ-C30: a quality-of-life instrument for use in international clinical trials in oncology. J Natl Cancer Inst 85:365-376. https://doi.org/10.1093/ jnci/85.5.365

19. Sprangers MAG, Groenvold M, Arraras JI, Franklin J, te Velde A, Muller M, Franzini L, Williams A, de Haes HC, Hopwood P, Cull A, Aaronson NK (1996) The European Organization for Research and Treatment of Cancer breast cancer-specific qualityof-life questionnaire module: first results from a three-country field study. J Clin Oncol 14:2756-2768. https://doi.org/10.1200/ JCO.1996.14.10.2756

20. Fayers P, Aaronson N, Bjordal K, Curran D, Groenvold M (2001) EORTC QLQ-C30 scoring manual, 9th edn. EORTC Study Group on Quality of Life, Brussels

21. Snyder CF, Smith K, Bantug E, Tolbert EE, Blackford AL, Brundage MD (2017) What do these scores mean? Presenting patientreported outcomes data to patients and clinicians to improve interpretability. Cancer 123:1848-1859. https://doi.org/10.1002/cncr. 30530
22. Koller M, Lorenz W (2002) Quality of life: a deconstruction for clinicians. J R Soc Med 95:481-488. https://doi.org/10.1258/jrsm. 95.10.481

23. Klinkhammer-Schalke M, Koller M, Wyatt JC, Steinger B, Ehret C, Ernst B, Hofstädter F, Lorenz W (2008) Quality of life diagnosis and therapy as complex intervention for improvement of health in breast cancer patients: delineating the conceptual, methodological, and logistic requirements (modeling). Langenbecks Arch Surg 393:1-12. https://doi.org/10.1007/s00423-007-0210-5

24. Grol R, Grimshaw J (2003) From best evidence to best practice: effective implementation of change in patients' care. Lancet 362:1225-1230. https://doi.org/10.1016/S0140-6736(03)14546-1

25. Joseph-Williams N, Elwyn G, Edwards A (2014) Knowledge is not power for patients: a systematic review and thematic synthesis of patient-reported barriers and facilitators to shared decision making. Patient Educ Couns 94:291-309. https://doi.org/10.1016/j. pec.2013.10.031

26. Wysham NG, Wolf SP, Samsa G, Abernethy AP, LeBlanc TW (2017) Integration of electronic patient-reported outcomes into routine cancer care: an analysis of factors affecting data completeness. JCO Clinical Cancer Informatics. https://doi.org/10.1200/ CCI.16.00043

Publisher's note Springer Nature remains neutral with regard to jurisdictional claims in published maps and institutional affiliations. 